stated predominant beverage. Body weight data were available in 216 controls and (pre-fluid overload) in 198 patients.

Results TLA was (median (IOR)) $118(80-175) \times 10^{3}$ Units in patients and $131(93-183) \times 10^{3}$ units in controls $(p=0.041)$ by Mann-Whitney): 9 (6-12)-fold and 9 (7-12)-fold higher respectively than the minimum specified by the above inclusion criteria. TLA, corrected for body wt was similar in patients and controls. Females with ALD consumed less total alcohol (absolute 85 (52-11) vs $136(94-193) \times 10^{3}$ units $(p<0.01)$ and corrected for wt $(p=0.04))$, and also, less beer $(p=0.001)$ but more wine $(p<0.001)$ and a trend towards more spirits $(p=0.166)$ over their lifetime than did males with ALD. However, female controls showed similar significant differences from male controls: lower TLA (both absolute $82(69-119)$ vs $145(108-193) \times 10^{3}$ Units) and corrected for body weight) and also, less beer, more wine and more spirits (all comparisons $\mathrm{p} \leq 0.001$ ).

Conclusion The higher total alcohol consumption in controls than in patients is more consistent with a threshold effect then a dose effect of alcohol for development of ALD. Male-female differences in consumption are independent of the presence of liver disease and therefore, are not evidence per se of increased female susceptibility to ALD, although the data do not exclude this.

Competing interests None declared.

\section{PTU-028 TRANSIENT ELASTOGRAPHY IN THE RISK STRATIFICATION OF PATIENTS WITH ABNORMAL LIVER FUNCTION TESTS}

doi:10.1136/gutjnl-2012-302514c.28

E J Britton, * R Saravanan, T Woodhouse, K Koss. Department of Gastroenterology, Macclesfield District General Hospital, Macclesfield, UK

Introduction Transient elastography (TE) (Fibroscan) is a well validated method for assessing liver fibrosis in a trial setting. Gastroenterology Department at Macclesfield DGH is one of the first centres in the UK to adopt Fibroscan examination into the diagnostic pathway of patients with abnormal LFT. This is the first publication of our data of unselected patients undergoing liver stiffness measurement (LSM) demonstrating practicalities and limitations of Fibroscan.

Methods Data were collected from 366 patients with abnormal LFT attending Gastroenterology Clinic from July 2010 to December 2011. Fibroscan examination was performed by two gastroentrology consultants and one specialist nurse. The cohorts of Fatty liver disease (FLD) and Alcoholic liver disease (ALD) were further analysed comparing fibrosis score from TE with clinical findings, laboratory blood tests, ultrasound imaging and liver biopsy.

Results Patients diagnosis were as follows: FLD 35.8\% ( $n=131)$, ALD $14.8 \%(n=54)$, Chronic hepatitis C $15.6 \%(n=57)$, PSC/PBC $7.7 \%(n=28)$, chronic hepatitis B $4.6 \%(n=17)$, haemochromatosis $2.2 \%(n=8)$, AIH $1.4 \%(n=5)$, chronic usage of Methotrexate $1.9 \%$ $(n=7)$. Additionally 42 healthy volunteers participated. The mean time duration for each successful scan was 173 (30-1363) seconds with $18 \%$ of patients requiring the $\mathrm{XL}$ probe due to central obesity. The fibrosis scores recorded for all patients scanned were F0-1 41\% $(\mathrm{n}=150)$, F2 6.3\% $(\mathrm{n}=23), \mathrm{F} 3 \mathrm{7.1} \%(\mathrm{n}=26)$ and F4 26\% ( $\mathrm{n}=95) .15 \%$ of scans were considered to be failed as LSM/IOR was $>33$, most prevalent in the FLD group $16.8 \%(n=22)$ and wasn't operator or probe dependent. Significant proportion of patients with liver fibrosis (F3-4) had no clinical signs of chronic liver disease. $80 \%$ of patients F3-F4 fibrosis demonstrated portal hypertension on imaging and $32 \%$ of patients in that group had low platelets
(3. The sensitivity and specificity of low platelets for significant fibrosis was $32 \%$ and $91 \%$ and for portal hypertension on imaging was $64 \%$ and $89 \%$ respectively. There was significant correlation between liver fibrosis scoring on TE and Metvir fibrosis scoring on biopsy in patients without high degree of inflammation. However, coexisting acute inflammation in some cases contributed to false positive results on Fibroscan.

Conclusion The Fibroscan technology has allowed rapid stratification of patients with chronic liver diseases. Significant proportion of patients (40\%) were appropriately reassured without need of undergoing liver biopsy. $20 \%$ of patients without stigmata of chronic disease and without conventional laboratory markers of liver fibrosis were diagnosed with liver cirrhosis. Early experience has shown difficulties of performing TE in patients with central obesity and FLD. Fibroscan finds its valuable role in the care pathway of patients with abnormal LFT.

Competing interests None declared.

\section{PTU-029 A NOVEL MRI PROTOCOL TO EXAMINE HAEMODYNAMIC COMPARTMENTS IN COMPENSATED LIVER CIRRHOSIS}

doi:10.1136/gutjnl-2012-302514c.29

${ }^{1} \mathrm{E} F$ Cox, ${ }^{*}{ }^{2} \mathrm{~A}$ Ghezzi, ${ }^{2} \mathrm{M}$ Patel, ${ }^{2} \mathrm{~A}$ Jackson, ${ }^{1} \mathrm{C}$ Costigan, ${ }^{2} \mathrm{M}$ W James, ${ }^{2} \mathrm{~S} D$ Ryder, ${ }^{1} \mathrm{P}$ A Gowland, ${ }^{2} \mathrm{G}$ P Aithal, ${ }^{1} \mathrm{~S}$ T Francis, ${ }^{2} \mathrm{~N}$ Guha. ${ }^{1}$ Sir Peter Mansfield Magnetic Resonance Centre, University of Nottingham, Nottingham, UK; ${ }^{2}$ NIHR Nottingham Digestive Diseases Biomedical Research Unit, Nottingham University Hospitals, Nottingham, UK

Introduction The hyperdynamic circulation in cirrhosis results from changes in the splanchnic, systemic, cardiac and renal compartments; it underpins the clinical consequences of portal hypertension. We present a novel MRI protocol that provides non-invasive measurement of these different haemodynamic compartments in a single assessment. Phase contrast (PC) MRI is a validated technique for the measurement of velocity (in $\mathrm{cm} / \mathrm{s}$ ), area, and hence flux (=velocity*area (in $\mathrm{ml} / \mathrm{s})$ ) in a given vessel. ${ }^{1}$ Here, we use PC-MRI, together with MR measures of cardiac function, to assess haemodynamics in early, compensated cirrhosis and matched healthy volunteers.

Methods Patients were studied from a prospective, longitudinal study of compensated cirrhosis (CC) (Child Pugh A) with age and sex matched healthy volunteers (HV). 20 CC patients (13M/7F, age $57 \pm 1$ y (mean \pm SEM), aetiology 2 ALD, 1 HBV, 7 HCV, 3 NAFLD, 1 PBC, 1 Haemochromatosis, MELD 0-10.6) and $20 \mathrm{HV}$ : 13M/7F, age $57 \pm 2$ y were included. PC-MRI data were collected for the superior mesenteric (SMA), splenic (SA), hepatic (HA), and renal (RA) artery and portal vein (PV). 15 phases were acquired across the cardiac cycle to calculate the mean area, velocity and flux for each vessel. Cardiac MR consisted of short axis cine images to measure left ventricular ejection fraction (EF) and PC-MRI of the aorta. All data were collected within $20 \mathrm{~min}$, with images acquired during short breath-holding (12-15 s).

Results In CC, the portal vein was dilated and had a reduction in velocity compared to the HV group (Abstract PTU-029 figure 1A). The SMA (Abstract PTU-029 figure 1B) and SA, showed vasodilation and increased flow velocity, and a resulting increase in flux in patients with CC compared to the HV. There was a trend for a reduction in RA velocity (Abstract PTU-029 figure 1C) and an increase in HA velocity in CC. The ejection fraction was significantly higher in CC compared with the HV (Abstract PTU-029 figure 1D). There was no change in aortic flux, velocity or area with CC. 

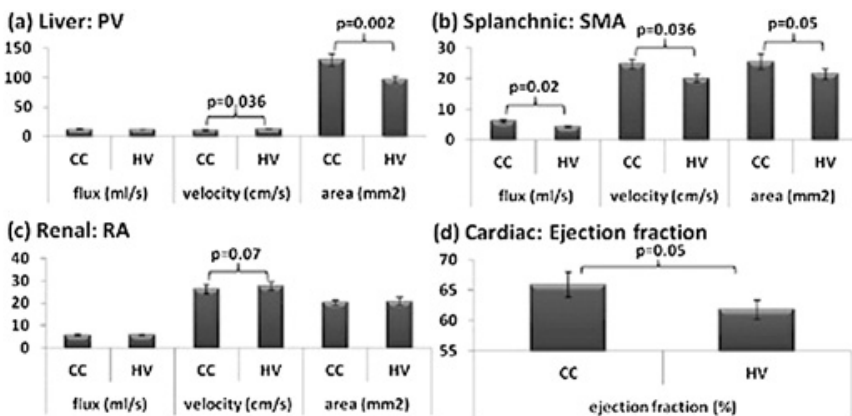

\section{Abstract PTU-029 Figure 1}

Conclusion Using a non-invasive MRI protocol, we have measured haemodynamics in four compartments contemporaneously in cirrhosis. The detection of significant changes in early cirrhosis, suggests this technique has potential to (a) study the evolution of portal hypertension with accompanying changes in splanchnic, renal and systemic circulation as well as (b) assess the haemodynamic response to novel therapeutic agents in cirrhosis.

Competing interests None declared.

\section{REFERENCE}

1. Debatin JF. Abdom Imaging 1998;23:485-95.

\section{PTU-030 SELECTIVE GUT DECOMTAMINATION REDUCES HEPATIC EXPRESSION OF TOLL-LIKE RECEPTOR (TLR) 4 AND DEVELOPMENT OF CIRRHOSIS BUT DOES NOT PREVENT DEVELOPMENT OF HEPATOCELLULAR CARCINOMA (HCC)}

doi:10.1136/gutjnl-2012-302514c.30

${ }^{1} \mathrm{~F}$ Mohamed, ${ }^{2} \mathrm{D}$ Dhar, ${ }^{1} \mathrm{~N}$ Shah, ${ }^{1} \mathrm{~N}$ Davies, ${ }^{1} \mathrm{~A}$ Habtesion, ${ }^{3} \mathrm{~T} \mathrm{~V}$ Luong, ${ }^{4} \mathrm{~A}$ Winstanley, ${ }^{5} \mathrm{~A}$ P Dhillon, ${ }^{3} \mathrm{~S}$ Oldedamink, ${ }^{1} \mathrm{R}$ P Mookerjee, ${ }^{1} \mathrm{R}$ Jalan. ${ }^{1}$ Hepatology, University College Hospital, London, UK; ' ${ }^{2}$ niversity College London, London, UK; ${ }^{3}$ Royal Free Hospital, London, UK; ${ }^{4}$ University College Hospital, London, UK; ${ }^{5}$ Pathology, Royal Free Hospital, London, UK; ${ }^{6}$ Hepatology, University College Hospital, London, UK

Introduction Recent studies suggest that TLR4 inhibition may prevent fibrosis in murine models. Chronic antigenic stimulation due to increased bacterial gut translocation leads to upregulation of hepatic TLR4 and this may lead to fibrosis, cirrhosis and HCC. The aims of this study were to determine whether gut decontamination with Norfloxacin prevents cirrhosis and HCC in rodent model of cirrhosis and HCC.

Methods 18 Fisher rats divided into three groups; 1st treated with DEN and NMOR (carcinogens). 2nd was treated with the same carcinogens + Norfloxacin from the beginning to 14 weeks (end of experiment). 3rd group was control. H\&E, reticulin and Immunohistochemistry were done also liver function and TNF- $\alpha$ were measured.

Results All the rats in 1st and 2nd groups developed HCC, the severity of which was not different between groups. With reticulin stain there was significantly reduced fibrosis in the group treated with Norfloxacin (score: $1(0-2))$ compared with the untreated group (score: $4(3-5))(p<0.03)$. The expression of TLR4 in both carcinogen groups in the background liver was significantly greater than control rats but lower in the Norfloxacin group compared with the untreated group $\left(p<0.0016^{* * *}\right)$. No difference inTLR4 expression was observed in the HCC nodules in both groups. There was a reduction of ALT $(p<0.05)$ and AST $(p<0.01)$ in the Norfloxacin treated comparing to DEN and NMOR group. There was a also reduction of TNF- $\alpha$ level in the Norfloxacin treated group.
Conclusion The results of this study suggest that selective decontamination of the gut may be a novel strategy to prevent cirrhosis probably by inhibiting hepatic TLR4 expression. In this model of cirrhosis and HCC, reduction of hepatic TLR4 does not prevent development of HCC suggesting that the mechanisms of its development are unrelated to severity of fibrosis and TLR4 related mechanisms.

Competing interests None declared.

\section{PTU-031 DOWNREGULATION OF THE VITAMIN B $_{\mathbf{1 2}}$ RECEPTOR IN FIBROLAMELLAR CARCINOMA OF THE LIVER: THE FIRST CONSISTENT MOLECULAR ABNORMALITY}

doi:10.1136/gutjnl-2012-302514c.31

${ }^{1} \mathrm{P}$ Johnson, ${ }^{*}{ }^{1} \mathrm{G}$ Baldwin, ${ }^{1} \mathrm{M}$ Teng, ${ }^{2} \mathrm{~B}$ O'Sullivan, ${ }^{3} \mathrm{~J}$ James, ${ }^{4} \mathrm{M}-\mathrm{A}$ Brundler, ${ }^{2} \mathrm{P}$ Taniere. ${ }^{1}$ School of Cancer Sciences, University of Birmingham, Birmingham, UK; ${ }^{2}$ Pathology, Queen Elizabeth Hospital, Birmingham, UK; ${ }^{3}$ Biosciences, University of Birmingham, Birmingham, UK; ${ }^{4}$ Pathology, Birmingham Children's Hospital, Birmingham, UK

Introduction Fibrolamellar hepatocellular carcinoma (FLHCC) is a rare tumour of young adults that is characterised by the presence of large tumour cells with strongly eosinophilic cytoplasm, surrounded by fibrous lamellae, in the absence of chronic liver disease. No consistent molecular abnormalities have been detected to date but an elevation of serum vitamin $B_{12}$ binding capacity has been described.

Methods Up to $80 \%$ of vitamin $\mathrm{B}_{12}$ (cobalamin), is stored in the liver after endocytosis via the transcobalamin receptor (CD320). We hypothesised that disruption to the transcobalamin receptor (hereafter referred to as CD320) might render the liver locally $B_{12}$ deficient and elicit a compensatory production of the serum $\mathrm{B}_{12}$ binding protein (TCN1). We sought a functional mutation of CD320 in 15 cases of FLHCC and 30 cases of conventional hepatocellular carcinoma (HCC), 10 cases with cirrhosis (C-HCC) and 20 cases with no cirrhosis (NC-HCC)) acting as control tissues. cDNA was synthesised using RNA purified from formalin fixed paraffin embedded (FFPE) tissues and primers were designed to amplify the region spanning exon $2 / 3$ across the mutation site.

Results Sequencing analysis showed $\sim 5 \%(1 / 18)$ mutation in NC HCC. However, $60 \%(9 / 15)$ of the FLHCC cohort did not show any amplification while $80 \%$ of NC-HCC (16/20) and C-HCC (8/10) tumours tested positive. The quality of purified mRNA was verified by amplifying for two different housekeeping genes, tubulin and $\beta$-glucuronidase. To determine the reason for non-amplification of CD320exon2/3, we sequenced the promoter and promoter regulator region of CD320 using DNA purified from FFPE tissue. Comparison of the sequences of $\mathrm{CD} 320(+)$ and $(-)$ representative samples showed no difference in their DNA sequence. Methylation analysis of the methylation sensitive promoter regulator region of NC-HCC, and FLHCC showed a correlation between the level of methylation and CD320 suppression in NC-HCC but not for FLHCC. To address this discrepancy, a second region of CD320, spanning exon3/4 was amplified. Interestingly, all the FLHCC samples were positive giving an amplification product for this region. Immunostaining for CD320 was in agreement with the exon 3/4 amplification data.

Conclusion Taken together, these results indicate that the expression of a non-functional splice variant (variant 2), which lacks the crucial triple glutamic acid repeat within the exon 2 necessary for the receptor's function, may contribute to the increased serum Vitamin $\mathrm{B}_{12}$ binding capacity in FLHCC.

Competing interests None declared. 